\title{
Tooth brushing with and without Dentifrice - A Comparative Study
}

\begin{abstract}
L Pathak
Abstract

Tooth brushing is such a familiar process that it is easy to forget how crucial it is to oral health and how important it is to brush properly. Twenty three patients were selected in the age-group of 30-40 having tooth brushing abrasion in the cervical region of at least three teeth and taught the Modified Stillman's Brushing Technique during a four week pre-test period. Plaque disclosing agent was used prior to and after brushing to educate the subjects. At the end of the pre-test period a complete oral prophylaxis was done to obtain a'zero' plaque score. The patient was asked to refrain from any oral hygiene procedure for next 48 hours and nonfibrous regular diet was advised. After 48 hours pre-brushing plaque scoring was recorded according to Turesky-Gilmore-Glickman Modification of the Quigley-Hein plaque index. A split mouth design was followed to record the post brushing plaque scores. Each time the patients were asked to brush on both upper and lower quadrants of one side for recording post-brushing plaque scores 'without dentifrice' first and then brush on the upper and lower quadrants of the other side for recording plaque scores' with dentifrice'. The three treatment groups viz. Pre-brushing, Post-brushing without dentifrice and Post-brushing with dentifrice when subjected to ANOVA confirmed that the Modified Stillman's Technique effectively removes plaque in both post-brushing situations $(p<0.01)$. The subjects showed a reduction in plaque levels in the Post-brushing trials as indicated by C.D. value (0.0179) ( $p<0.05)$. It can be derived from the statistical analysis that if properly done tooth brushing removes plaque effectively when used with or without dentifrice.
\end{abstract}

Keywords: Toothbrush, Tooth brushing, Modified Stillman's Technique.

\section{Introduction}

Procedures for control of supragingival plaque are as old as recorded history of Mankind. Charaka Samhita stressed the importance of tooth brushing and oral hygiene in ancient India. The first patent for a manual toothbrush was issued to Wadsworth in the middle of the 19th Century. A manual toothbrush is a device composed of a shaft with either natural or synthetic bristles at one end intended to remove adherent plaque and food debris from the teeth surfaces to reduce tooth decay ${ }^{1}$. Unbeknown to most users the toothbrush is a medical device regulated under the Food, Drug and Cosmetic Act and Regulated Laws $1992^{2}$. Till now no such toothpaste currently available is accepted by The American Dental Association as ideal for prevention of gingivitis. Indeed tooth brushing with toothpaste was found to be responsible for a high degree of cervical abrasion $^{3}$. Many variables like brushing technique, duration and frequency of brushing and type of particular filament stiffness may cause cervical abrasion to a large extent ${ }^{4-7}$.

\section{Address of Correspondence:}

Dr. Liza Pathak, MDS (Periodontology), Reader, Department of Periodontology, Regional Dental College,Guwahati, India, E-mail: liza0368@gmail.com
If used correctly brushing can remove plaque from tooth surface without a dentifrice ${ }^{8}$. Cleaning efficacy of different brushing techniques have been reported in many studies ${ }^{9}$. It is observed that Modified Stillman's Tooth brushing has been the technique of choice for a very few trials. This technique is seen to take care of the teeth and gingiva of the individuals who already developed cervical abrasion due to improper brushing technique. The present study aims to evaluate and compare the cleaning efficacy of the Modified Stillman's Tooth brushing Technique in the individuals who are not exposed to proper brushing and has already developed cervical abrasions.

\section{Methodology}

For the purpose of the study twenty three patients who had never received any professional dental care were recruited from the fourth grade employees of the University Hospital.

\section{Exclusion Criteria}

Every patient was selected only in the age group of $30-40$ and those who had periodontal therapy in the past were excluded from the study. Those having crowding, faulty restorations and any systemic disease were excluded. All the twenty three patients presented at least three teeth together with cervical abrasion. 


\section{Armamentarium used in the study}

A detailed medical and dental history was taken from each patient participating in the study. Mouth mirrors, tweezers, kidney tray, dappen dish, mouth mask, gauze strips, ultrasonic scaler, airotor handpiece, rubber cups and pumice powder, medium textured toothbrushes, dentifrice containing one thousand PPM of fluoride, acrylic models for demonstration of brushing technique, looking mirrors and disclosing solution (erythrocin dye) was used for the study.

\section{Pre-Test Period}

At the beginning of the study written consent was obtained from all the patients. A complete oral prophylaxis was given to each of them to eliminate the interference of stain and calculus deposits in the grading of plaque. A four week pre-test period followed the oral prophylaxis during which the subjects were taught the importance of the oral physiotherapy programme by incorporating the prescribed tooth brushing technique. Each patient was taught the "Modified Stillmans Technique" of tooth brushing on acrylic models. They were given looking mirrors and plaque disclosing solutions to see the plaque removal prior to and after brushing. The brushing technique was demonstrated in the following way.

\section{Modified Stillman's Technique}

\section{A. Position the brush}

1. Filaments. Direct the filaments apically.

2. Place side of the brush on the attached gingival.

B. Strokes

1. Place to flex the filaments. The sides of the filaments are pressed lightly against the gingiva. The gingiva will blanch under pressure.

2. Angle the filaments. Turn the handle by rotating the wrist so that the filaments are directed at an angle of approximately $45^{\circ}$ with the long axis of the tooth.

3 . Activate the brush with a slight vibratory motion. The tips of the filaments should touch the gingival with a light pressure.

4. Roll and vibrate the brush while working slowly down over the gingival and tooth. Make some of the filaments reach interdentally.

C. Replace Brush for Repeat strokes.

\section{Repeat Strokes for each two quadrants.}

Steps A through C was repeated in two quadrants (upper and lower) of the same side at a time. The time allotted was 2 minutes and 3 minutes for each side (right and left) for different sessions. The two quadrants of the same side was scored after brushing for 2 mins. and 3 mins. and also with and without dentifrice.

E. Position brush for anterior lingual and palatal surfaces the long, narrow way and repeat the strokes as in $B$.

\section{F. Occlusal Brushing}

1. Place brush on occlusal surface of molar teeth with filament tips pointed into the occlusal pits at a right angle. The handle should be parallel with the occlusal surface. The toe of the brush should cover the distal groove of the most posterior tooth.

2. Vibrate the brush in a slight circular movement, while maintaining the filament tips on the occlusal surface. Press moderately so that the filaments do not bend but go straight into the pits and fissures.

3. Move brush to the premolar area, overlapping previous brush position repeat 1 and 2 steps.

Experimental Procedure

At the end of the pre-test period i.e. on day 0 the patient was called.

Day 0

Every subject was comfortably seated on a dental chair. Rubber cup polishing was done with pumice powder. Then the subject was asked to keep the disclosing solution in mouth for one minute. Any amount of plaque disclosed was removed by scaling and polishing until 'zero' plaque score was obtained. Thereafter the subject was asked to refrain from any oral hygiene procedure without fibrous diet for next 48 hours.

Day 1

After 48 hours the subject reported to the clinic. The subject was asked to swiss the solution (1:50 dilution) inside mouth for 2 minutes. This was followed by a thorough rinse with plain water to remove any excess of the disclosing solution. A split-mouth design was followed and Pre-brushing plaque score was recorded in all the four quadrants according to Turesky-Gilmore-Glickman modification of the Quigley-Hein plaque index as described before. This established the amount of plaque formed during the 48 hours period while the subject refrained from any oral hygiene measures to serve as baseline control. 
After the pre-brushing scoring the subject was given a toothbrush (medium textured) and was asked to brush the teeth for two minutes on the two quadrants of the same side (both maxillary and mandibular) without any dentifrice. Following brushing the plaque was disclosed and post-brushing plaque data 'without dentifrice' for two minutes was recorded. Thereafter the subject was asked to put an approximately $1 / 3 \mathrm{rd}$ of an inch length of dentifrice on the bristles and brush on the other two remaining quadrants in the opposite side for two minutes. The post-brushing plaque score 'with dentifrice' for two minutes was recorded.

After a gap of another week the subjects were recalled and the experimental procedure was repeated from Day-0 to Day-1 but this time the test quadrants were reversed for recording the data in the post brushing scoring for the same duration i.e. two minutes.

Again after a gap of one week the subjects were recalled.Procedure from Day-0 to Day-1 was repeated but this time the subjects brushed for three minutes duration for each quadrants of the same side. The post-brushing data recorded for both with and without dentifrice. After one week the subjects were recalled for repeat sessions from Day-0 to Day-1.The brushing time was three minutes and the post-brushing data was recorded by reversing the quadrants. All the data were collected by a single examiner (self).

\section{Results and observations}

Twenty three subjects participated in this clinical study. A splitmouth design was followed to record all the data. A plaque index was calculated for each subject as follows:

$$
\mathrm{PI}=\frac{(\text { total plaque score) }}{(\text { no.of tooth surfaces) }}
$$

The pre-brushing PI = the amount of plaque measured before tooth brushing

The post-brushing PI (with toothpaste and without toothpaste) $=$ the amount of plaque measured after tooth brushing.

A mean plaque index (MPI) was calculated as follows:

\section{MPI $=$ Mean \pm S.E.(Standard Error)}

The experimental data obtained were subjected to statistical analysis. The mean age of all the subjects was 33 years. For the purpose of comparison between the plaque levels at different situations the following were considered as different treatment groups:
- Pre-brushing plaque levels at 2 mins.

- Pre-brushing plaque levels at 3 mins.

- Post-brushing with dentifrice plaque levels at 2 mins.

- Post-brushing with dentifrice plaque levels at 3 mins.

- Post-brushing without dentifrice plaque levels at 2 mins.

- Post-brushing without dentifrice plaque levels at 3 mins.

Table I shows the distribution of plaque scores recorded as per Turesky-Gilmore-Glickman modification of the Quigley-Hein plaque index. Table II shows a decrease in the plaque levels for all the post-brushing MPIs. Table III shows that the t-values in all the treatment groups bear no statistical significance when brushing time was increased from 2 mins. to 3 mins. Table IV shows the values of three treatment groups after ANOVA was done. This analysis confirmed that Modified Stillman's Technique effectively removes plaque in both post-brushing treatment groups $(\mathrm{p}<0.01)$.

Table $\mathrm{V}$ shows the various plaque levels and their p-values. Here treatment groups shown as having same superscript do not differ significantly. The post-brushing data in both situations as indicated in the C.D. value $(0.0179)$ is significant $(\mathrm{p}<0.05)$.

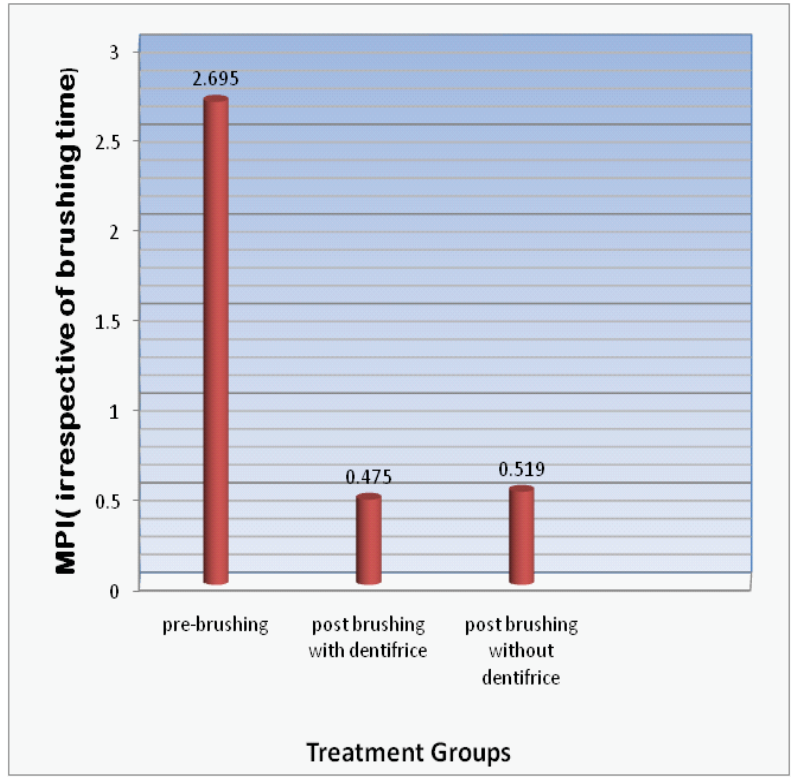




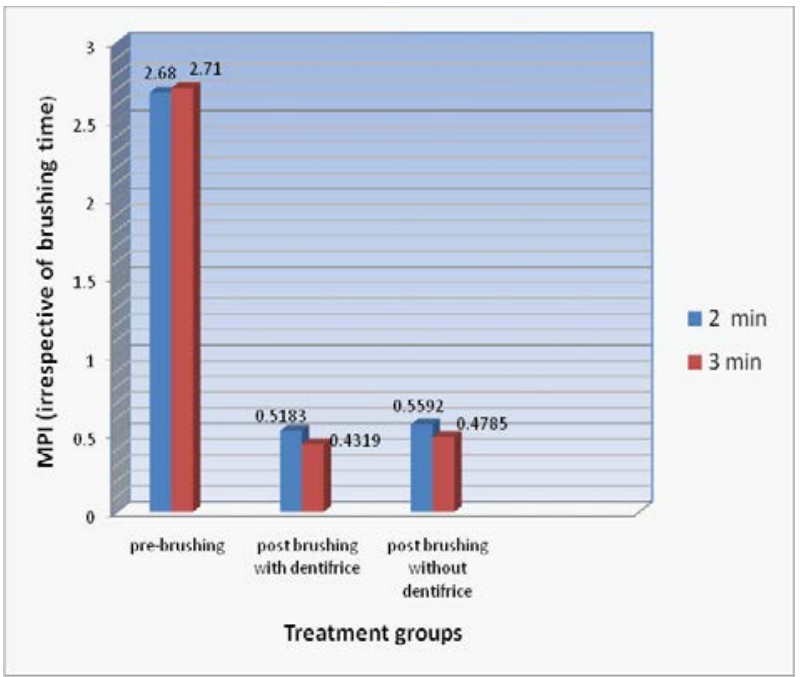

Table I : Distribution of Subjects by Plaque Score

\begin{tabular}{|c|c|c|c|c|c|c|}
\hline Score & \multicolumn{2}{|c|}{ Pre -Brushing } & \multicolumn{2}{|c|}{ Post -Brushing (With) } & \multicolumn{2}{|c|}{ Post -Brushing(Without) } \\
\hline & 2 & 3 & 2 & 3 & 2 & 3 \\
\hline $\mathbf{0}$ & 0 & 0 & 764 & 833 & 735 & 790 \\
\hline $\mathbf{1}$ & 154 & 130 & 476 & 441 & 482 & 459 \\
\hline $\mathbf{2}$ & 892 & 886 & 104 & 70 & 127 & 95 \\
\hline $\mathbf{3}$ & 1337 & 1393 & 0 & 0 & 0 & 0 \\
\hline $\mathbf{4}$ & 295 & 265 & 0 & 0 & 0 & 0 \\
\hline $\mathbf{5}$ & 10 & 14 & 0 & 0 & 0 & 0 \\
\hline
\end{tabular}

Table II : Split Mouth Data Comparison of Mean Plaque Index for the Two Treatment with Controlled Baseline

\begin{tabular}{|l|l|l|l|l|l|l|}
\hline & \multicolumn{2}{|c|}{$\begin{array}{c}\text { Pre -Brushing } \\
\text { (Controlled Baseline) }\end{array}$} & \multicolumn{2}{c|}{$\begin{array}{c}\text { Post -Brushing } \\
\text { (With Dentifrice) }\end{array}$} & \multicolumn{2}{c|}{$\begin{array}{c}\text { Post -Brushing } \\
\text { (Without Dentifrice) }\end{array}$} \\
\hline Timing & 2 & 3 & 2 & 3 & 2 & 3 \\
\hline Mpi & 2.680 & 2.711 & 0.5183 & 0.4319 & 0.5592 & 0.4785 \\
& \pm 0.065 & \pm 0.061 & \pm 0.031 & \pm 0.023 & \pm 0.034 & \pm 0.028 \\
\hline
\end{tabular}

Table III : Values Shown After T-Test for the three Different Situations

\begin{tabular}{cccc}
\hline \multicolumn{4}{c}{ t-Value } \\
\hline \multicolumn{4}{c}{ Treatment Groups } \\
t-Valu & $\begin{array}{c}\text { Pre-Brushing } \\
\text { (Controlled Baseline) }\end{array}$ & $\begin{array}{c}\text { Post-Brushing } \\
\text { (With Dentiffice) }\end{array}$ & $\begin{array}{c}\text { Post-Brushing } \\
\text { (Without Dentifrice) }\end{array}$ \\
& 0.23 & 1.538 & 1.059 \\
d.f. & 44 & & \\
& & 44 & \\
Statisti & NS & & \\
Signific: & & NS & \\
& & & \\
\hline
\end{tabular}

Table IV : Reduction in Plaque Levels by Modified Stillman's Tooth brushing Technique (Anova)

\begin{tabular}{|l|c|c|c|c|}
\hline $\begin{array}{l}\text { Sources of } \\
\text { variation }\end{array}$ & d.f. & S.S. & M.S. & F \\
\hline Treatment & 2 & 2996.61147 & 148.30577 & 1631.526 \\
\hline Error & 273 & 24.81269 & 0.0909 & \\
\hline Total & 275 & 321.427766 & & \\
\hline
\end{tabular}

d.f $=$ Degrees of freedom

$(\mathrm{p}<0.01)$

$\mathrm{S} . \mathrm{S}=$ sum of squares

M.S = Mean sum of squares

$\mathrm{F}=$ Variance Ratio

Table V : Comparison of Plaque Levels of the Three Treatment Groups And Their P-Value

\begin{tabular}{|c|c|c|c|}
\hline Treatment Groups & \multicolumn{2}{|c|}{ Plaque levels } & P-Value \\
\hline $\begin{array}{l}\text { Pre -Brushing } \\
\text { (Controlled Baseline) }\end{array}$ & $2.695^{\mathrm{a}}$ & \pm 0.063 & $\mathrm{P}<0.05$ \\
\hline $\begin{array}{l}\text { Post -Brushing } \\
\text { (With Dentifrice) }\end{array}$ & $0.4751^{\circ}$ & \pm 0.027 & $(C . D .=0.0871)$ \\
\hline $\begin{array}{l}\text { Post -Brushing } \\
\text { (Without Dentifrice) }\end{array}$ & $0.5188^{\circ}$ & \pm 0.031 & \\
\hline
\end{tabular}

\section{N.B. : Treatment groups having same superscript do not differ significantly}

\section{Discussion}

Promotion of oral health and prevention of oral diseases have been the focus of oral hygiene practice since its inception. In our country social and economic conditions have deprived a large section of people an adequate oral health practice. There are many tooth brushing studies which will remove plaque effectively without harmful effect on hard \& soft tissues ${ }^{10-12}$. The Modified Stillman's tooth brushing technique fairly works well for patients with tooth brushing abrasions as well as anatomically normal gingiva. In our endeavor to study the effectiveness of this brushing technique we evaluated the treatment groups in different situations. The data showed that all the 23 individuals accumulated a minimum level of plaque (score 1). The score of 3 was obtained in maximum no. of pre-brushing situations both at $2 \& 3$ minutes. A small no. of individuals accumulated the maximum plaque level (score 5) during 48 hours of oral hygiene abstinence. 
It was noted that the post brushing treatment groups (with or without dentifrice) did not differ significantly as seen in table III. The emphasis is to be given on the method how the brush is employed and not on the abrasive action of the dentifrice. In the present study a split mouth design once followed whereby two quadrants (upper and lower) were brushed alternatively with and without dentifrice to observe the reduction in plaque levels. In both the situations a significant reduction in plaque levels was observed $(p<0.05)$. The Modified Stillman's brushing technique seems to be an ideal oral hygiene practice which does not necessitate the use of toothpaste always.

\section{Conclusion}

Tooth brushing is a simple mechanical measure which can be easily taught as individual home oral therapy programme .It is necessary to find out which technique the patient can perform best .It is useless to insist on a technique which the patient finds difficult and ends up having cervical abrasions. Indeed, manual dexterity does not necessarily go with intellect and some of the brightest patient's prove to be extremely incompliant when it comes to cleaning the teeth .Regular guidance and encouragement are essential for people to develop the necessary skill. Most of the people engage in faulty brushing which in the course of time results in tooth brushing abrasions. Modified Stillman's Technique of brushing may be incorporated as the preferred technique of choice for the overall maintenance of health of gingiva and the teeth.

\section{References}

1. American Academy of Periodontology, Committee Report :The Toothbrush and Methods of Cleaning the teeth $1920 ; 42: 193$.

2.US Department of Health and Human Services, Public Health Service, Food and Drug Admininstration Center of Devices and Radiological Health :Everything you always wanted to know about medical device requirements and weren't afraid to ask,4th edn. Publication FDA 1992;92-2173.
3. Kitchin P :The prevalence of Tooth root exposure and the relation of such exposure to the degree of Abrasion in different Age classes J Dent Res 1941;20:565-581.

4. Saxton CA and Cowell CR :Clinical Investigations of The Effects of Dentifrices on Dentine Wear at The Cementoenamel Junction.JADA1981;102:38-43.

5. Sangnes $G$ :Traumatisation of Teeth and Gingiva Related to Habitual Toothcleaning. Review Article.J.Clin.Periodontol 1976;3:94-103.

6. Frandsen A :Mechanical Oral Hygiene Practices In :Dental Plaque Control Measures and Oral Hygiene Practices,ed. Loe H and Kleinman, DV IRL Press 1986 ;39-71.

7. Eid MA and Talic YF :A Clinical Trial on The Effectiveness of Professional Tooth brushing Using Dentifrice and Water :Odontostomatol Trop1991;14(2):9-12.

8. Gift HC :Current Utilisation Patterns of Oral Hygiene Practices.In : Loe H.Kleinman DV.ed.Dental Plaque Control Measures and Oral Hygiene Practices.Oxford:IRL Press 1986;39-71.

9. Dyer D, Addy M, Newcombe RG :Studies in Vitro of Abrasion by Different Manual Toothbrush Heads and a Standard Toothpaste 2000;27:99-103.

10. Jepsons :The Role of Manual Toothbrushes in Effective Plaque Control :Advantages and Limitations .In :Lang NP, Attstrom R, Loe H, eds. Proceedings of The European Workshop on Mechanical Plaque Control. Berlin :Quintessence Publishing Co.Inc.1998:72-84.

11. Poyato M, Segura JJ. Effecto del aprendizaje de las tecnicas de hygiene oral sobre el indice de placa de los estudiantes de 'Higiene Buco-dental'. Arch Odontoestomatol Prev Commune 2000;16:177-182.

12. Perry Dorothy A, Mcdowell James and Goodies Harold E:Gingival Microcirculation Response to Tooth brushing Measured by Laser Doppler Flowmetry 1997: Vol 68 No.10:990-995. 